\title{
Post-traumatic Stress Disorder and Psychological and Social Adjustment among Libyan Who Participated in 17th of February Revolution: A Structural Equation Modeling Analysis
}

\author{
Fariha Omar M. Elfaydi, Ibrahim Mikail, and R. A. H. Kirembwe
}

\begin{abstract}
Post-traumatic stress disorder (PTSD) is an increasingly recognized and potentially preventable condition. Certain factors, especially the severity of the trauma, perceived lack of social support and pre-traumatic dissociation have been associated with its development. In recent years, a more robust evidence base regarding the management of individuals involved in traumatic events has emerged. This evidence suggested that people might suffer traumatic disorder, immediately after a traumatic event, or appear after several months. This has a negative effect psychological and social order for the survivors of trauma. The purpose of this study is to investigate the hypothesized causal relationships between post-traumatic stress disorder (PTSD) and psychological social adjustment among Libyans who participated in the revolution of 17th of February. The researcher collected data from a sample of 215 participated and injured in the Libyan revolution of February 17 used a self-reported questionnaire. Structural equation modeling was used to establish the relationship between traumatic disorder and social adjustment It was found that the post-traumatic stress disorder (PTSD) negatively affect psychological and social adjustment. Moreover, the researcher also as was hypothesized found that religious commitment alleviated level of (PTSD) and smoothen the psychological and social adjustment .The study revealed that there is a positive relationship between post-traumatic stress disorders with the lack of psycho-social compatibility. It also revealed that some of the injured in the fighting fronts suffer from social isolation.
\end{abstract}

Index Terms-Post-traumatic stress disorder (PTSD), psychological adjustment, social adjustment, religious commitment, structural equation modeling (SEM).

\section{INTRODUCTION}

Post-traumatic disorder has been defined in many ways by different scholars defending on schools of thought. However, all these different definition asserted that posttraumatic disorder is a mental disorder and anxiety developed as a result of exposure a terrifying event or ordeal in which grave physical harm occurred or was threatened. Family members of victims also can develop the disorder. The post-traumatic disorder can occur in people of any age, including children and adolescents. More than twice as many women as men experience. It is believed that people who exposed to trauma as a result of bad event might experience post-traumatic disorder. Among common events that might lead to post-traumatic disorder are earthquakes, hurricanes, wars, rape, car accidents, aircraft accident, and

Manuscript received March 4, 2015; revised June 1, 2015.

The authors are with Faculty of Major Languages Studies, Universiti Sains Islam Malaysia (USIM) Bandar Barn Nilai, 71800 Nilai, Negeri Sembilan, Malaysia (e-mail: Mikail@usim.edu.my, fefe1980f@gmail.com). collision of trains. These event might cause people expose to an incident very stressful beyond human experience and beyond their imagination which eventually may cause traumatic disorder. The thoughts and memories of scary and disturbing nightmares. These disorders may persist for a long period of time if cares were not seriously taken [1].

The post traumatic disorder was first discovered in 1970 immediately after the Vietnam War, when the symptoms of this disorder began appear on the American soldiers participated in the war. After nine to thirty months from the end of the war, and the soldiers return to their country, and the completion of their military service, many of them showed the symptoms of mental disorders. Despite the fact that more than a quarter of a century had passed on the war, but a large group of those soldiers are still suffering from the symptoms of this chronic disorders. [2] Traumatic disorder has been classified into various categories such as anxiety disorder, phobia, Obsessive-compulsive, and posttraumatic stress disorder. A number of empirical studies has found the strong relationship between both types of traumatic-disorder and psychological disorder. For example. [3] in his empirical study discovered a strong relationship between post-traumatic stress disorder and psychological disorder such as stress, anxiety, suicide, homicide and mental problem. These psychological problems are not only individual problems but also the problems of government and society at large.

\section{EXPLANATORY THEORIES FOR (PTSD)}

Many of the views of previous studies on post-traumatic stress disorder (PTSD) focused on biological orientation interpretation of the problem. The biological interpretation of post-traumatic disorder mainly based on genetic factors. According to this approach people that people who suffered from this chronic disorder belonging to the families of the infected individuals with mental disorders precedent, and genes may mediate between neurotic personality traits, and post-traumatic stress disorder which eventually operate on a person's willingness to injury Post Traumatic Stress. [4] concluded that genetics play a role in the filming of (PTSD).Using the genes of interest to the serotonin system, because encoding and transmission of serotonin is a genetic region that may modulate emotional response to traumatic events.

On the other hand, psychodynamic Approach based on Freud interpretation of post-traumatic stress disorder suggested that the shock of birth that accompanied with sense of newborn suffocated was the major cause of posttraumatic disorder. The approach emphasized that shock of 
the birth was the first anxiety experience that newborn baby experienced in his/her own life which eventually developed to post-traumatic disorder (PTSD). [5] also indicated that the depression and post-traumatic stress disorder dramatically increasing in response to the negative effects such as rape, car accident and war. As was previously highlighted, many psychoanalytic researchers found that human exposure to trauma in early life is a serious factor for the emergence of mental disorders and chronic illnesses.

On the other hand, behavioral approach contended that environments plays a significant role in emergence or disappearance of post-traumatic disorder. Environment according to this approach is a major determinant of human behavior, because it teaches human to acquire a conditional fear in response to conditional response. A person traumatized by the war, trying to escape (avoidance) of stimuli that remind shocked, because these stimuli caused him mental problem and pains of remembrance.

Moreover. [6] In clinical results , exposure to traumatic events makes a person's brain to produce physiological changes in reaction to that event in order to face the external threat or to avoid it.

Cognitive approach also believed that mental disorders caused by irrational thinking about the Life events, the shock demolished the concept of security. As a result, that irrational thinking becomes real life perspective of the infected person.

Notwithstanding, studies investigated the impact of social factor such as social supports on post-traumatic stress disorder. Moreover [7] found that the Israeli soldiers who participated in the Lebanese war, but received the high levels of social attribution, showed low symptoms of posttraumatic stress disorder.

This finding is supported by another theory which explained that (PTSD) when exposed individual to shock, his/her brain receives a lot of information, which it would be interpreted wrongly due to outweigh the nervous energy and lack of human experience to absorb it and deal with it positively. The information processing will be then volatile, leading to distorted, and remain active in his/her memory. Therefore, an affected person with Post Traumatic Stress recover the shock by disturbing nightmares. [8] emphasized that there is an increase in brain activity during the face of trauma in the emotional networks including front amygdala, and the anterior cingulate cortex, which normally appear in the form of a sharp Emotions, because of the severity of the shock.

\section{StAtement of The Problem AND Its SignificANCE}

This studies aims to investigate the post-traumatic stress disorder experienced by Libyans who participated in 17 of February revolution. Given what passed by Libya in political, economic and social changes, and happened between protesters and Gaddafi's battalions during the revolution which lead to uses of heavy weapons. Also the torture and murder, or execution of innocent citizens who they were eventually found in mass graves after the end of the revolution. All these events had a negative impact on the behavior of returnees from combats, and who need to great efforts for re-adapt into the society. The bloody confrontations which led to thousands of death, violence and mental warrant this study.

Because most of the protesters were universities Students, teachers, and shops' owners who are all young age, so they were physically and psychologically unprepared to face this kind of heavy battle which eventually led to more than 5000 deaths and 3500 injured. Therefore, helping the injured, and prepared them psychologically, socially and professionally, to start their new life is very crucial.

\section{SAmPle AND the Statistical Methods USED}

This study was conducted on 215 Libyans participated in the 17 of November revolution. The sample were randomly selected from three distinctive regions, to obtain a representative sampling. The researcher constructed two types of instrument to measure the traumatic disorder and social compatibility of the target population due to unavailability of an appropriate scale precisely measure what the researcher attempts to measure. The first instrument consisted of 28 items which was categorized into four dimensions, namely, Skirting, Repetition, Astonishing, Emotional, And religiosity and Physical turmoil. On other hand, the social compatibility instrument consisted of 22 items which eventually digested into four distinctive factors. These factors are family Compatibility, Personal Compatibility, Emotional Compatibility, and Compatibility with others respectively.

The internal consistency of both instrument was tested using Cronbach alpha. The result of analysis suggested that the value of Cronbach's alpha for traumatic disorder ranged between .70 to .92 , while its value for social compatibility ranged between .77 to .95 . These value suggested that the instruments are reliable since the value of their internal consistency is high and thus they can be used for any meaningful academic .The researcher used the discriminant validity, convergent validity to test the validity of the used instrument. Moreover, Exploratory Factor Analysis was used to digest the item of the scale into the latent variables. The researcher used Exploratory factor Analysis (EFA) instead of Principal Component Analysis to extract the factors from the common variance only. Unlike Principal Component Analysis which extracted factors from common variance, unique variance and error variance, EFA is only extract factor from common variance alone. Therefore, this technique give EFA superiority of PCA because the significant of the contribution of each item into the factor can be exactly identified [9].

Furthermore, Structural Equation Modeling was also used to establish the casual relation among the targeted constructs namely post-traumatic disorder, psychological disorder and social adjustment. This is because Statistical method able to study the dependent variables, and several independent variables simultaneously and in parallel.

The Fig. 1 is illustration of the model which used to establish the relationship between different components of the study. The figure showed the causal relationship between Post-traumatic stress disorder and Psychological and social compatibility 


\section{RESUlt OF ANALysis}

The proposed model was tested using Structural Equation model as was previously highlighted.. The researcher used AMOS 16 to test the proposed model and the result of analysis suggested that the model was fit. A number of indices were used to test the fitness of the model; among the fit indices that were used are chi-square with it respective degree of freedom and p-value. In addition to these absolute values . the researcher also used relative indices value such as Goodness of fit index (GFI),Adjusted Goodness of fit (AGFI) index, Incremental fit index (IFI),Comparative fit index (CFI), Tucker-Lewis index (TLI) Root-mean-square of approximation (RMSEA) (see Fig. 1).

Although, the chi-square was statistically significant which indicated that the model was not fit well. The other indices suggested that the model was fit. However, since chi-square is very sensitive to the sample size, the researcher used other indicators to determine the significant of the model and its fitness. The results of the analysis suggested that there is casual relationship between posttraumatic stress disorder on one hand and psychological and social adjustment $(r=1.00)$. This result indicated that the post-traumatic disorder affects psychological and social adjustment. Furthermore, the study showed emotional disorder is the major factor of post-traumatic disorder $(r$ $=0.76)$ followed by avoidance $(r=0.61)$ and repetition $(r$ $=0.60)$.

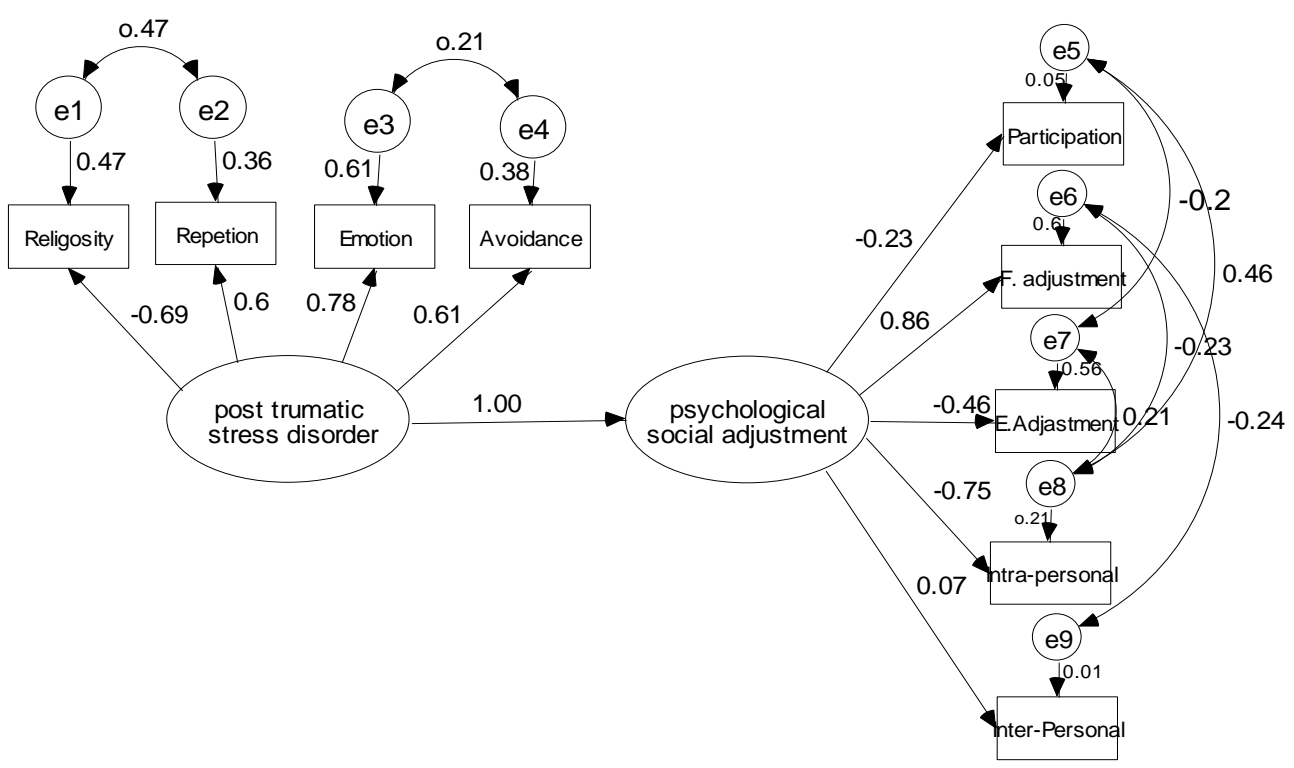

Fig. 1. Structural modeling (1) of post-traumatic disorder.

Also, the study indicated native relationship between religiosity and post-traumatic stress disorder $(r=-0.69)$. This proves that the person troubled Tries Avoid shocking memories .He also suffers from emotional disorders and physical abuse can be observed. This suggests that people who have suffered post-traumatic stress disorder, People who have a weakness in religious commitment. And appeared relationship between repetition and disorder after a positive shock $(r=-0.60)$.

Furthermore, there is also positive relationship between psychosocial compatibility and family support $(r=0.86)$. The positive relationship between psychological adjustment and social compatibility with others $(r=0.07)$. This result confirms that the person who enjoys consensus psychosocial. It is compatible with himself and others.

Moreover, there is also negative relationship between psychological and social compatibility, and social participation, $(r=-0.23)$ and intrapersonal compatibility, $(r=$ -0.75). The results also indicate that there is a negative relationship between the psychological and social compatibility, emotional $(r=-0.46)$.

\section{DISCUSSION}

The current study examined the effects of involvement in war on low psychological adjustment among the Libyan participated in the 17 of February revolution. More specifically, it tested a model in which post-traumatic disorder contributed to psychological disorder.

The results of the study demonstrate that participated in the war have enormous impact on psychological disorder and disability to cope with social and psychological. [10] demands. These findings support previous studies documenting the long-term impact of war captivity on both PTSD. [11] other studies have shown that war-related PTSD casualties report greater impairments in spousal intimacy and they are more prone to outbursts of rage and aggression, and decrease in sexual desire So focused the peoples Which has suffered from wars to study post-traumatic disorder, And the speed of treatment so Can victims Adapt to normal life After returning from the fighting, And get rid of obsessive-compulsive disorder, and Nightmares nagging Nightmares nagging associated with war and Live in peace with their families

\section{REFERENCES}

[1] E. sherin, "Post traumatic stress disorder. The neurobiological impact of psychological trauma," PhD, University of Miami, USA, pp. 263278, 2011.

[2] R. G. Parsons and K. J. Ressler, "Implications of memory modulation for post-traumatic stress and fear disorders," US National Library, vol. 16, pp. 146-153, 2013.

[3] R. Smith, H. Batric, and T. Perrin, "Exposure to war and the reactions of the mothers in the psychological adjustment of children from 
Bosnia and Herzegovina," Journal Psychology Psychiatry for Children the Number the Third London, vol. 23, pp. 186-202, 2003.

[4] L. Eric, T. Micheal, and M. Robert, "Co morbidity of (PTSD ) and genetic makeup," Journal Psychology Is Natura, pp. 334-347, 2010.

[5] Z. Oken and S. Wehb, "The relationship between post-trauma disorder and the level of cortisol when veterans," Journal Psychiatry, vol. 27, May 2013.

[6] C. Neylan and G. Mueller, "Imaging in post traumatic stress disorder repetition of trauma Co morbidity psychological interaction," PhD. University of Florida, vol. 67, no. 3, pp. 296-303, 2010.

[7] J. Anton, B. Michiel, and H. Smit, Increase the Anterior Cingulated Cortex and Hippocampus Activation in (PTSD) During Encoding Negative Words, Oxford University, vol. 2, pp. 181-187, 2011.

[8] F. Michael, W. Carol, M. Gutjrie, and B. Donald. "Cognitivebehavioral psychotherapy for (PTSD) and results of clinical trials," Journal of Child Psychology And Psychiatry, vol. 21, pp. 217-220, 2011.

[9] P. Kline, An Easy Guide to Factor Analysis USA, Second Edition, pp. 116-120, 2013.

[10] C. Beckham and S. D. Moore, "Interpersonal hostility and violence in Vietnam combat veterans with chronic posttraumatic stress disorder:
A review of theoretical models and empirical evidence," Aggression and Violent Behavior, vol. 5, pp. 451-466, 2000.

[11] J. M. Cook, D. S. Riggs, R. Thompson, and J. C. Coyne, "Posttraumatic stress disorder and current relationship functioning among world war II ex-prisoners of war," Journal of Family Psychology, vol. 18, pp. 36-45, 2004.

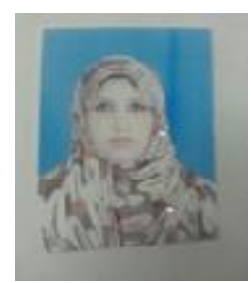

Fariha Omar M. Elfaydi was born in 1980, in Benghazi, Libya. She got a master's in psychology in 2009. She worked as a faculty member at the University of Benghazi for four years.

She worked as the head of the Department of Psychology for a year.

She has participated in a conference on drugs and a conference on psychological health inside Libya. And now she is in the final stage of the doctoral

thesis in mental health. 